OPEN ACCESS

Edited by:

Massuo Jorge Kato,

University of São Paulo, Brazil

Reviewed by:

Qi Wu,

Sichuan Agricultural University, China

Chaofu Lu,

Montana State University,

United States

Dimitre Alexandrov Ivanov, University of Western Ontario, Canada

*Correspondence: Jeongim Kim jkim6@ufl.edu

Specialty section: This article was submitted to Plant Metabolism and Chemodiversity,

a section of the journal

Frontiers in Plant Science

Received: 20 August 2019

Accepted: 09 January 2020

Published: 05 February 2020

Citation:

Zhang D, Song YH, Dai R, Lee TG and Kim J (2020) Aldoxime Metabolism is Linked to Phenylpropanoid Production in Camelina sativa.

Front. Plant Sci. 11:17. doi: 10.3389/fpls.2020.00017

\section{Aldoxime Metabolism Is Linked to Phenylpropanoid Production in Camelina sativa}

\author{
Dingpeng Zhang ${ }^{1}$, Yeong Hun Song ${ }^{1}$, Ru Dai ${ }^{1}$, Tong Geon Lee ${ }^{1,2,3}$ and Jeongim Kim ${ }^{1,3^{*}}$ \\ 1 Horticultural Sciences Department, University of Florida, Gainesville, FL, United States, ${ }^{2}$ Gulf Coast Research and \\ Education Center, University of Florida, Wimauma, FL, United States, ${ }^{3}$ Plant Molecular and Cellular Biology Graduate \\ Program, University of Florida, Gainesville, FL, United States
}

Plants produce diverse secondary metabolites. Although each metabolite is made through its respective biosynthetic pathway, plants coordinate multiple biosynthetic pathways simultaneously. One example is an interaction between glucosinolate and phenylpropanoid pathways in Arabidopsis thaliana. Glucosinolates are defense compounds made primarily from methionine and tryptophan, while phenylpropanoids are made from phenylalanine. Recent studies have shown that the accumulation of glucosinolate intermediate such as indole-3-acetaldoxime (IAOx) or its derivatives represses phenylpropanoid production via the degradation of phenylalanine ammonia lyase (PAL) functioning at the entry point of the phenylpropanoid pathway. Given that IAOx is a precursor of other bioactive compounds other than glucosinolates and that the phenylpropanoid pathway is present in most plants, we hypothesized that this interaction is relevant to other species. Camelina sativa is an oil crop and produces camalexin from IAOx. We enhanced IAOx production in Camelina by overexpressing Arabidopsis CYP79B2 which encodes an IAOx-producing enzyme. The overexpression of AtCYP79B2 results in increased auxin content and its associated morphological phenotypes in Camelina but indole glucosinolates were not detected in Camelina wild type as well as the overexpression lines. However, phenylpropanoid contents were reduced in AtCYP79B2 overexpression lines suggesting a link between aldoxime metabolism and phenylpropanoid production. Interestingly, the expression of PALs was not affected in the overexpression lines although PAL activity was reduced. To test if PAL degradation is involved in the crosstalk, we identified F-box genes functioning in PAL degradation through a phylogenetic study. A total of 459 transcript models encoding kelch-motifs were identified from the Camelina sativa database. Among them, the expression of CsKFBs involved in PAL degradation is up-regulated in the transgenic lines. Our results suggest a link between aldoxime metabolism and phenylpropanoid production in Camelina and that the molecular mechanism behind the crosstalk is conserved in Arabidopsis and Camelina.

Keywords: Camelina sativa, aldoxime, phenylpropanoids, auxin, PAL degradation 


\section{INTRODUCTION}

As sessile organisms, plants produce diverse secondary metabolites which play crucial roles in their adaptation to surrounding conditions. Although each metabolite is produced through its respective biosynthetic pathway, plants orchestrate multiple biosynthetic pathways simultaneously. Recent studies have shown the influence of glucosinolate intermediates on phenylpropanoid production (Hemm et al., 2003; Kim et al., 2015; Kim et al., 2019).

Glucosinolates are defense compounds and over 100 kinds of glucosinolates have been found in plants (Agerbirk and Olsen, 2012). Phenylpropanoids such as lignin and flavonoids are made from phenylalanine through the phenylpropanoid pathway and are involved in diverse aspects of plant growth and development (Vogt, 2010; Fraser and Chapple, 2011). Although phenylpropanoids and glucosinolates are produced through their own biosynthetic pathways, a link between the two pathways was identified from Arabidopsis studies.

Arabidopsis mutants with defects in the formation of the core structure of glucosinolates show defects in both phenylpropanoid production and glucosinolate biosynthesis (Hemm et al., 2003; Kim et al., 2015). For instance, Arabidopsis ref5 and ref2 mutants were originally isolated due to their phenylpropanoid deficiency phenotypes (Ruegger and Chapple, 2001). However, genetic mapping studies revealed that ref5 and ref2 have mutations in CYP83B1 and CYP83A1, respectively (Hemm et al., 2003; Kim et al., 2015). Both CYP83A1 and CYP83B1 are involved in the oxidation of aldoximes, which is required for the core structure of glucosinolates (Naur et al., 2003). Although both genes have broad substrate specificities toward aldoximes, CYP83A1 functions mainly in aliphatic glucosinolate biosynthesis whereas CYP83B1 has higher activity toward indole-3acetaldoxime (IAOx). Further biochemical studies have revealed that the accumulation of aldoximes or their derivatives, rather than a deficiency of glucosinolates or the accumulation of IAA, is responsible for the repression of phenylpropanoid production in ref5 and ref2 (Hemm et al., 2003; Kim et al., 2015). Studies with ref5 suppressor revealed that Arabidopsis mediator subunit 5 (MED5) is involved in the link between the glucosinolate and phenylpropanoid biosynthesis pathways. The steady state phenylalanine pools in ref5 mutants were higher than that in the wild type suggesting that the inhibition may occur at or downstream of PAL in the phenylpropanoid biosynthetsis pathway (Kim et al., 2015). A transcriptome study with a set of glucosinolate-deficient mutants identified that there are MED5-dependent and MED5-independent mechanisms behind the crosstalk, and that the increased levels of aldoximes up-regulate kelch-domain containing F-box genes functioning in the ubiquitination of PALs (Kim et al., 2019).

The aldoximes are made from various amino acids by the cytochrome P450 monooxygenase 79 family members (CYP79) (Irmisch et al., 2013; Irmisch et al., 2015; Luck et al., 2017; Bhalla et al., 2018; Sørensen et al., 2018; Dhandapani et al., 2019). Arabidopsis thaliana has five functional CYP79s. CYP79F1 and CYP79F2 convert homomethionine to aliphatic aldoximes and CYP79A2 produces phenylacetaldoxime from phenylalanine
(Wittstock and Halkier, 2000; Hansen et al., 2001; Chen et al., 2003). CYP79B2 and CYP79B3 function redundantly to convert tryptophan to IAOx (Mikkelsen et al., 2000; Zhao et al., 2002). In particular, IAOx is a precursor of indole-glucosinolates, IAA, and camalexin (Zhao et al., 2002; Sugawara et al., 2009; Klein et al., 2013) (Figure 1A), therefore overexpression of AtCYP79B2 increases the production of IAA and indole glucosionolates (Zhao et al., 2002). Since aldoximes are precursors of various metabolites in addition to glucosinolates (Irmisch et al., 2013; Irmisch et al., 2015; Luck et al., 2017; Bhalla et al., 2018; Sørensen et al., 2018; Dhandapani et al., 2019) and the phenylpropanoid pathway is conserved in most plants, it is possible that a similar interaction occurs in other plant species. This study aims to elucidate a connection between IAOx metabolism and the phenylpropanoid pathway in Camelina sativa (Figure 1A).

Camelina sativa is an annual Brassicaceae oilseed crop. Due to its high levels of oil and unique oil composition with over $90 \%$ unsaturated fatty acid in its seeds, it is a valuable industrial oil crop for making jet fuel and biodiesel (Kagale et al., 2014). In addition, its seed meal is used as animal feed because of its residual essential fatty acids (Malik et al., 2018). Camelina produces glucosinolates and phenylpropanoids including sinapine (Russo and Reggiani, 2012). Since glucosinolates and sinapine limit the use of Camelina flours in animal diet due to their antinutritional properties (Russo and Reggiani, 2012), it is of great interest to manipulate these secondary metabolites. Given that $C$. sativa produces glucosinolates and camalexin from aldoximes (Browne et al., 1991; Klein et al., 2013), it is likely that aldoxime metabolism and the phenylpropanoid pathway coexist in C. sativa. To examine the impact of altered aldoxime metabolism on the phenylpropanoid pathway in $C$. sativa, we enhanced the IAOx production in $C$. sativa by overexpressing Arabidopsis CYP79B2 and evaluated its influence on plant growth and phenylpropanoid production. Our data suggest that the links between indole aldoxime metabolism and phenylpropanoid production found in Arabidopsis also occur in other Brassicaceae such as C. sativa.

\section{MATERIALS AND METHODS}

\section{Plant Materials and Growth Conditions}

Camelina sativa cv. Celine and Arabidopsis thaliana Col-0 were used as wild-type plants. Plants were grown at $22^{\circ} \mathrm{C} \pm 1{ }^{\circ} \mathrm{C}$ with 16-h light/8-h dark photoperiod. For seedlings grown on MS plates, the seeds were sterilized with $20 \%$ (v/v) bleach containing $0.005 \%$ triton X-100 for $10 \mathrm{~min}$. After being washed with water multiple times, the seeds were cold-treated at $4^{\circ} \mathrm{C}$ for 3 days, and then were planted on MS medium containing $2 \%$ sucrose and $1.2 \%$ agar. For soil-grown plants, the seeds were directly planted on soil after 3 days of cold treatment at $4^{\circ} \mathrm{C}$.

\section{Generation of Transgenic Plants Overexpressing AtCYP79B2 and CsKFB50}

For Camelina AtCYP79B2 overexpression lines, the published 995-AtCYP79B2 binary vector that carries AtCYP79B2 under the 


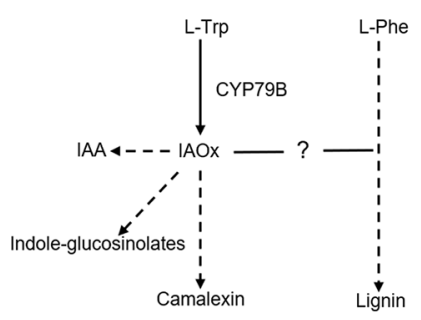

D

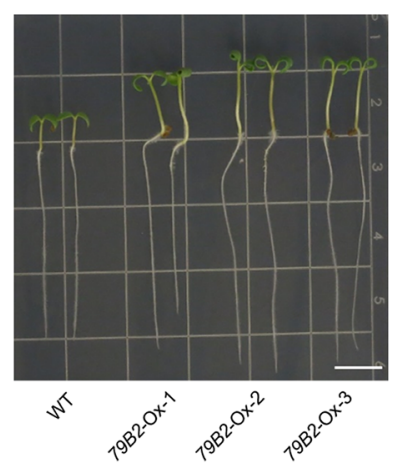

C

E

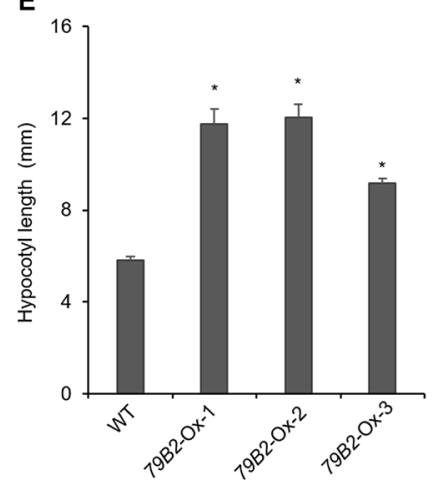

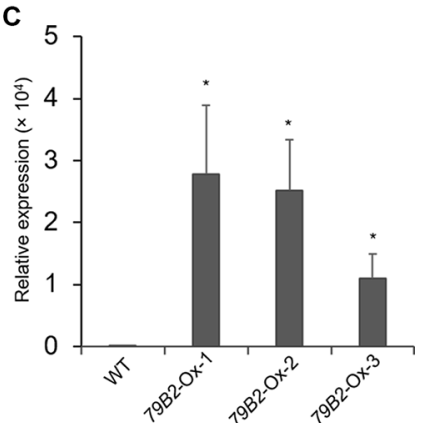

F

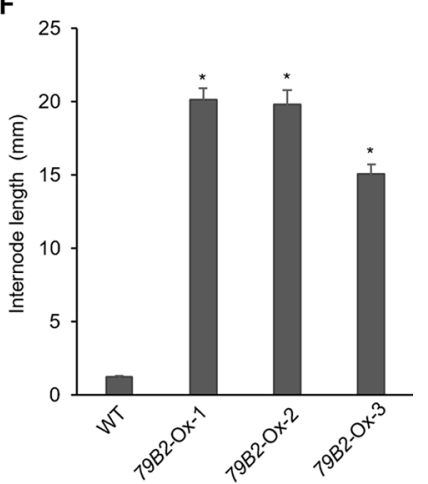

FIGURE 1 | Overexpression of AtCYP79B2 in Camelina results in characteristic morphologicalphenotypes. (A) A proposed link between IAOx metabolism and the phenylpropanoid pathway in Camelina. (B, C) Expression of AtCYP79B2 in three independent transgenic lines, 79B2-Ox-1, 79B2-Ox-2, and 79B2-Ox-3, was measured by semiquantitative RT-PCR (B) or quantitative RT-PCR (C). Total RNA was prepared from 14-day-old Camelina leaves grown on soil and CsACTIN was used as control. In (C), data represent mean \pm standard error (SE) from four independent biological replicates. (D) Representative 7-day-old Camelina plants grown on MS plate were photographed. Bar $=1 \mathrm{~cm}$. (E, F) Hypocotyl length of 7-day-old seedlings (E) and the internode length between the second leaf and the third leaf (F) were measured. 14-day-old plants grown on soil were used for the internode measurement. In (E, F), data represent mean \pm standard error (SE) ( $n=16)$.

* represents $P<0.05$ (two-tailed student's t-test) when compared with wild type.

CaMV 35S promoter was used for Camelina transformation (Kim et al., 2015). To generate a Camelina sativa Csa05g091560 overexpression construct, the open reading frame of Csa05g091560 was amplified by PCR using the primer pair of P19 and P20 and CDNA prepared from 6-day-old seedlings (Table S4). The amplified PCR product was cloned into a Gateway entry vector pCC1155, a modified version of pDONR221, and the resulting Csa05g091560 entry vector was recombined with destination vector pCC0995 (Weng et al., 2010), generating the 35S:Csa05g091560 construct. The intact ORF of Csa05g091560 in the 35S:Csa05g091560 construct was confirmed by sequencing before it was introduced into plants.

The constructs were introduced into Arabidopsis or Camelina plants by Agrobacterium-mediated flower dipping methods as previously described (Lu and Kang, 2008). The cultured Agrobacterium tumefaciens (strain GV3101) harboring the constructs was suspended in 5\% sucrose containing $0.01 \%$ (v/v) Silwet L-77 (PhytoTech, S7777). The flowers of mature plants were dipped in the Agrobacterium solution for $30 \mathrm{~s}$, and then the plants were kept for $24 \mathrm{~h}$ in the dark. To screen transformants, 10 -day-old plants were sprayed with $0.2 \%$ Basta (Rely 280, BASF, NJ) and surviving T1 lines were confirmed with the expression of AtCYP79B2 or Csa05g091560.

\section{Measurement of Hypocotyl and Internode Length}

Seven-day-old T4 generation Camelina seedlings grown on MS plates were used for hypocotyl measurement. All of the transgenic lines used for phenotyping were confirmed for the presence of constructs either by spraying Basta or PCR for BAR gene in the vector (Weng et al., 2010).

Two-week-old soil-grown Camelina plants were used for the internode measurement. Sixteen samples $(N=16)$ were measured for both experiments and significance was tested with a two-tailed student's t-test.

\section{Metabolite Analysis}

For phenylpropanoid analyses, fresh samples were frozen in liquid nitrogen and stored at $-80^{\circ} \mathrm{C}$ until extraction. Metabolites were extracted with $50 \%(\mathrm{v} / \mathrm{v})$ methanol at $65^{\circ} \mathrm{C}$ for $1 \mathrm{~h}$. After centrifugation at $10,000 \times \mathrm{g}$ for $10 \mathrm{~min}$, the supernatant was collected for HPLC analysis. Ten $\mu \mathrm{l}$ of extracts was analyzed on an UltiMate 3000 HPLC system (ThermoFisher Scientific, MA) equipped with an autosampler cooled to $10^{\circ} \mathrm{C}$ and a diode array detector (DAD). The compounds were separated on an Acclaim ${ }^{\mathrm{TM}} 120 \mathrm{C} 18$ column 
(75 mm $\times 3 \mathrm{~mm} ; 2.2 \mu \mathrm{m})$ coupled with a C18 guard column (10 $\mathrm{mm} \times 3 \mathrm{~mm} ; 5 \mu \mathrm{m})$ (ThermoFisher Scientific, MA), with mobile phases consisting of solvent A; $0.1 \%$ formic acid (v/v) in water and solvent B; acetonitrile, and the following gradient: $5 \%-20 \%$ increase of solvent B at 0-6 $\mathrm{min}, 20 \%-95 \%$ increase of solvent B at $6-8.5 \mathrm{~min}$, and $95 \%$ solvent $B$ at $8.5-10 \mathrm{~min}$. The column was equilibrated for $5 \mathrm{~min}$ between injections. The flow rate was 0.7 $\mathrm{ml} \mathrm{min}{ }^{-1}$ and the column temperature was $40^{\circ} \mathrm{C}$. The contents of chlorogenic acid and sinapoylmalate were quantified based on the peak area at 325 and $330 \mathrm{~nm}$, respectively, and chlorogenic acid (Sigma, MO, C3878) and sinapic acid (Sigma, MO, D7927) were used for the external standards. For glucosinolate analyses, frozen plant samples were extracted with $50 \%(\mathrm{v} / \mathrm{v})$ methanol at $65^{\circ} \mathrm{C}$ for one and a half hours. The relative levels of indole-3ylmethyl glucosinolate (I3M) were quantified based on the peak area at $229 \mathrm{~nm}$ (Lee et al., 2012). Soluble metabolite analyses were performed with three biological replicates and statistical significance was tested with a two-tailed student's t-test.

\section{Purification of IAA}

IAA analysis was performed as described by Novák et al. (2012) with slight modifications. Twenty $\mathrm{mg}$ of each sample was homogenized and extracted in $1 \mathrm{ml}$ of sodium phosphate buffer $\left(50 \mathrm{mM}, \mathrm{pH} 7.0,4^{\circ} \mathrm{C}\right)$ containing $0.1 \%$ diethyldithiocarbamic acid sodium salt. $\left[{ }^{13} \mathrm{C}_{6}\right]$-IAA (5 pmol per sample) was added as an internal standard. The plant extracts were incubated at $4^{\circ} \mathrm{C}$ for $60 \mathrm{~min}$ with continuous shaking and then centrifuged at $16,300 \times \mathrm{g}$ at $4^{\circ} \mathrm{C}$ for $15 \mathrm{~min}$. After centrifugation, the sample $\mathrm{pH}$ was adjusted to $\mathrm{pH} 2.7$ with concentrated hydrochloric acid, and the extracts were purified using solid-phase extraction on Oasis ${ }^{\mathrm{TM}}$ HLB columns $(1 \mathrm{ml} / 30$ mg, Waters, MA). The columns were conditioned with $1 \mathrm{ml}$ methanol and $1 \mathrm{ml}$ water, and equilibrated with $1 \mathrm{ml}$ sodium phosphate buffer ( $\mathrm{pH}$ 2.7). After sample application, the column was washed with $4 \mathrm{ml}$ of $5 \%$ methanol in water and eluted with $4 \mathrm{ml}$ of $80 \%$ methanol. The eluate was evaporated to dryness in vacuo and stored at $-20^{\circ} \mathrm{C}$ until further analysis.

\section{Quantitative Analysis of IAA}

All samples were analyzed by LC-MRM-MS (liquid chromatography multiple reaction monitoring mass spectrometry). The evaporated samples were dissolved in $100 \mu \mathrm{l}$ of mobile phase (35\% methanol containing $0.1 \%$ formic acid) prior to mass analysis using a TSQ Altis Triple Quadrupole MS/MS system with ion funnel connected to a Vanquish Horizon ultra-high performance liquid chromatography (UHPLC) system equipped with an Accucore C18 column, $3 \times 50 \mathrm{~mm}, 2.6 \mu \mathrm{m}$ (Thermo Scientific, MA). MRM parameters of IAA and labeled IAA (precursor $m / z$, fragment $m / z$, radio frequency (RF) lens, and collision energy) were optimized on the TSQ Altis Triple Quadrupole MS using direct infusion of the authentic standards. A binary gradient of $0.1 \%$ formic acid in water and $0.1 \%$ formic acid in methanol was used as mobile phases A and B, respectively. The gradient profile was: 35\% solvent B from 0-5 min and 35\% to $95 \%$ solvent B from 5-6 min, and the flow rate was $0.25 \mathrm{ml} \mathrm{min}^{-1}$. Afterwards, the column was washed with $95 \%$ methanol for $5 \mathrm{~min}$ and re-equilibrated to initial conditions for $5 \mathrm{~min}$. The mass spectrometer conditions were as follows: the spray voltage was applied at $3,200 \mathrm{~V}$ in the positive mode, and sheath gas, aux gas, and sweep gas were set at 50, 10, 0 (arb unit), respectively. Ion transfer tube and vaporizer temperatures were set at $200^{\circ} \mathrm{C}$ and $40^{\circ} \mathrm{C}$, respectively. For MRM monitoring, both Q1 and Q3 resolutions were set at 0.7 FWHM with CID gas at 1.5 mTorr. The scan cycle time was at $0.8 \mathrm{~s}$. Quantification was obtained by MRM of the precursor $\left([\mathrm{M}+\mathrm{H}]^{+}\right)$and the appropriate product ion using optimal RF value and collision energy for the various diagnostic transitions shown in Table S1. The analysis was done with four replicates and statistical significance was tested with a two-tailed student's t-test.

\section{PAL Activity}

The PAL activity assay was performed as described with some modifications (Kim et al., 2015). Crude proteins were extracted with protein extraction buffer (0.1 M Tris ( $\mathrm{pH} 8.3$ ), 10\% glycerol, and $5 \mathrm{mM} \mathrm{DTT}$ ) After extraction, protein content was measured by Bradford protein assay with Bradford reagent (Sigma, MO, B6916) following the manufacturer's protocol. Bovine serum albumin (BSA, ThermoFisher Scientific, MA) was used as a standard. For the PAL activity assay, $150 \mu \mathrm{l}$ of protein extract was mixed with $4 \mathrm{mM}$ phenylalanine in a total of $500 \mu \mathrm{l}$ reaction solution (0.1 M Tris ( $\mathrm{pH} 8.3$ ), 10\% glycerol, $5 \mathrm{mM} \mathrm{DTT}$ ) and incubated at $37^{\circ} \mathrm{C}$ for $90 \mathrm{~min}$. The reaction was stopped by adding $50 \mu \mathrm{l}$ of acetic acid and the reaction product was extracted with $750 \mu \mathrm{l}$ of ethylacetate. The mixture was centrifuged at $10,000 \times g$ for $5 \mathrm{~min}$ and $600 \mu \mathrm{l}$ of supernatant was transferred into microcentrifuge tubes and dried in Vacufuge plus (Eppendorf, NY). The final extract was dissolved in $90 \mu \mathrm{l}$ of $50 \%$ methanol and analyzed using HPLC. PAL activity assays were performed with three biological replicates and statistical significance was tested with a two-tailed student's t-test.

\section{RNA Extraction and Quantitative RT-PCR}

Total RNA was extracted with RNeasy Mini Kit (QIAGEN, MD, 74904). Three $\mu \mathrm{g}$ of total RNA was treated with DNase (Invitrogen, MA, AM1907) following the manufacturer's protocol, and then $1 \mu \mathrm{g}$ of RNA was used for the first-strand cDNA synthesis using reverse transcription kit (ThermoFisher Scientific, MA 4368814). Quantitative PCR was performed with SYBR Green PCR master mix (ThermoFisher Scientific, MA) in StepOnePlus Real-Time PCR system. The specific primers are listed in Table S4. The following primers were used: AtCYP79B2, P1 and P2; AtTUBULIN, P47 and P48; CsACTIN, P3 and P4; CsKFB1-like, P98 and P99; CsKFB20-like, P9 and P10; CsKFB39-like, P100 and P101; CsKFB50-like, P102 and P103; CsPAL-like1, P121 and P122; CsPAL-like2, P125 and P126. For semiquantitative RT-PCR, $0.5 \mu \mathrm{l}$ cDNA was used as a template. PCR reactions were performed in a $20 \mu$ l volume mix containing Taq 2x Master Mix (NEB, M0270S) and primers (Table S4). P1 and $\mathrm{P} 2$ were for AtCYP79B2 while P3 and P4 were for CSACTIN.

\section{Identification of C. sativa Kelch-Motif Orthologs Using Hidden Markov Models}

The C. sativa annotated protein dataset was obtained from NCBI (https://www.ncbi.nlm.nih.gov/genome/annotation euk/Camelina_sativa/101).Putative orthologs of Arabidopsis 
genes were identified by using a hidden Markov model search (HMMER3.1b2;http://hmmer.org). The seed alignment model (PF01344) from Pfam (https://pfam.xfam.org; data access on Apr. 19, 2018) was used to identify canonical proteins of C. sativa. $10^{-1} e$-value cutoff was applied. No manual curation of $C$. sativa sequences and screening of conserved motifs outside the kelch-motif were performed. In order to increase confidence that true orthologs were detected, a reciprocal BLAST search of the predicted $C$. sativa proteomes was performed using full-length Arabidopsis sequences. BLAST searches were performed using tools provided at NCBI (https://www.ncbi.nlm.nih.gov), and TAIR (http://www. arabidopsis.org). All the C. sativa proteins identified as matching Arabidopsis kelch-motif proteins are detected with an e-value of 0.0 by BLAST. Alignments were performed using the MUSCLE tool (muscle 3.8.31; Edgar, 2004 for the Linux platform. Maximum likelihood (ML) phylogenetic analysis of the protein sequence alignment was performed using RAxMLHPC-PTHREADS-SSE3 (version 8.2.3; Stamatakis, 2014 for the Linux platform using the VT model of amino acid substitutions and gamma distribution parameters estimated by the software. One thousand bootstraps were performed. Phylogenetic trees were visualized and edited in Geneious R10.2.2 (http://www.biomatters.com). The ML tree annotated by RAxML was provided in Newick format.

\section{Identification of C. sativa CYP79B2 and KFB50 Sequences}

For CYP79B2 and KFB50 amino acid alignments, their putative homologies in C.sativa were identified by using the whole sequence of Arabidopsis proteins with NCBI (https://www. ncbi.nlm.nih.gov) blast tools. The alignments were constructed in T-coffee website (http://tcoffee.crg.cat/apps/tcoffee/index. html), and were visualized in The Sequence Manipulation Suite (https://www.bioinformatics.org/sms/index.html). For CYP79B2 phylogenetic analysis of the protein sequence, the ML method and Poisson model were used in MEGA-X based on ClustalW alignment. Bootstrap with 1,000 replicates was performed to show the tree topology.

\section{RESULTS}

\section{The Overexpression of AtCYP79B2 Increases Auxin Content and Affects Plant Growth and Development in C. sativa}

CYP79B family converts tryptophan to IAOx in Arabidopsis. Seven CYP79B homologs that are highly similar to those of Arabidopsis thaliana CYP79B2 (AtCYP79B2) and its paralog AtCYP79B3 were identified using the Camalina sativa protein database (Table 1). Four of them (Csa10g002730, Csa12g003200, Csa12g002890, and Csa11g003080) show over 96\% amino acid sequence identity with AtCYP79B2 whereas the other three homologs (Csa7g052430, Csa16g044070, and Csa9g086670) are more related to AtCYP79B3 (Table 1). Considering their conserved domains and high sequence homology (Figure S1), it is likely that CsCYP79Bs have biochemical activities similar to that of AtCYP79Bs. To increase IAOx production in C. sativa, Camelina transgenic lines overexpressing AtCYP79B2 were generated (Figure 1). Among a series of AtCYP79B2 overexpression lines, three independent lines showing high expression of AtCYP79B2 were selected for further study (Figures 1B, C).

Interestingly, Camelina plants overexpressing AtCYP79B2 display characteristic morphological phenotypes such as curled down cotyledons and elongated hypocotyls and internodes that were previously observed in plants containing increased levels of auxin (Figures 1D-F and Figure S2, Delarue et al., 1998; Zhao et al., 2002; Kim et al., 2007; Kim et al., 2015). Indeed, the level of IAA in the transgenic lines is higher than that of wild type plants (Figure 2A). It thus appears that the overproduced IAOx in Camelina is redirected to the production of IAA as observed in Arabidopsis.

\section{The Increased IAOx Production Negatively Impacts the Phenylpropanoid Production in C. Sativa}

Arabidopsis plants overexpressing CYP79B contained increased indole glucosinolates (Zhao et al., 2002). However, we were not able to detect indole glucosinolates in young seedlings or old leaves of both Camelina wild type and all three overexpression lines (Figure 2B). To test whether increased IAOx metabolism affects phenylpropanoid production in Camelina, soluble phenylpropanoid contents in seedlings, cotyledons, and mature leaves were assessed. Methanol extract from Camelina leaves gave four recognizable peaks and chlorogenic acid was a major phenylpropanoid as shown in Figure 3A. The levels of chlorogenic acid and the other phenolic compounds were substantially reduced in the AtCYP79B2 overexpression lines compared to wild type (Figure 3 and Figure S3). This suggests a link between aldoxime metabolism and phenylpropanoid production in Camelina. The production of chlorogenic acid from phenylalanine requires a series of enzymes. PAL is the first enzyme of the phenylpropanoid pathway that converts phenylalanine to cinnamic acid. The transgenic lines display approximately $50 \%$ of wild-type PAL activity (Figure 4A), which may contribute to the reduced levels of phenylpropanoids in the overexpression lines.

TABLE 1 | Protein sequence identity of Camelina CYP79Bs compared with Arabidopsis CYP79Bs.

\begin{tabular}{lccc}
\hline \multirow{2}{*}{ Gene name } & Chromosome & \multicolumn{2}{c}{ Identity (\%) } \\
\cline { 3 - 4 } & & AtCYP79B2 & AtCYP79B3 \\
\hline Csa10g002730 & 10 & 97 & 85 \\
Csa12g003200 & 12 & 96 & 85 \\
Csa12g002890 & 12 & 96 & 84 \\
Csa11g003080 & 11 & 96 & 84 \\
Csa7g052430 & 7 & 84 & 93 \\
Csa16g044070 & 16 & 85 & 93 \\
Csa9g086670 & 9 & 78 & 86
\end{tabular}




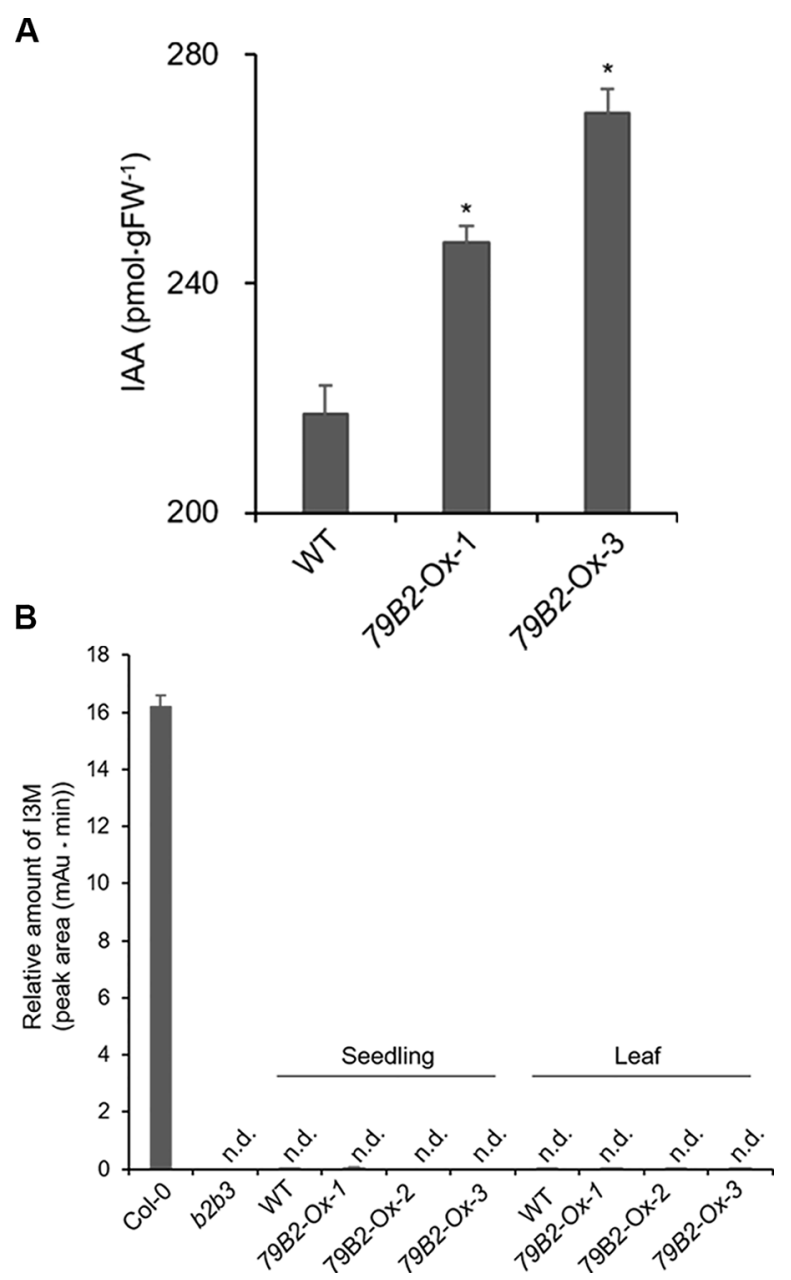

FIGURE 2 | The Levels of IAA are Increased but Indole Glucosiniolate is Deficient in Camelina Transgenic Plants Overexpressing AtCYP79B2. (A) Free IAA contents in the Camelina overexpression lines and wild type. Six-day-old seedlings grown on MS medium were collected for IAA measurement. Data represent mean \pm SE of four independent experiments. * represents $P<0.05$ (two-tailed student's t-test) when compared with wild type. (B) Relative levels of indole-3-ylmethyl glucosinolate (I3M) in C. sativa and $A$. thaliana. The levels of I3M were measured from 6-day-old Camelina seedlings grown in MS medium or leaves from 8-week-old plants grown on soil. Arabidopsis Col-0 and cyp79b2 cyp79b3 (b2b3) were used as positive and negative controls. Relative contents of I3M are shown based on peak areas of HPLC chromatograms at the retention time where I3M in Col-0 was eluted. Data represent mean \pm SE of three independent experiments. n.d., not detected.

\section{The Identification of Kelch-Domain Containing F-Boxes (KFB) in C. sativa}

PAL activity can be regulated at various levels including transcriptional and posttranslational regulations. We first assessed the levels of PAL expression in the transgenic lines. There are four PALs: PAL1, PAL2, PAL3, and PAL4 in Arabidopsis. We identified eight potential PAL homologs in Camelina. Csa06g039710, Csa05g016810, and Csa04g050600 show over 97\% sequence identity with Arabidopsis PAL1, whereas Csa06g027730, Csa04g039260, and Csa09g061550 are more related to Arabidopsis PAL2. Similarly, Csa01g011180 and Csa15g012820 are Arabidopsis PAL4 homologs with 94\% sequence identity. Interestingly, we were not able to find PAL3 homologs from the NCBI protein database.

Two sets of primers were designed to detect the transcript abundance of these eight genes. CsPAL-like1 detects PAL1 and PAL2 homologs (Csa06g039710, Csa05g016810, Csa04g050600, Csa06g027730, Csa04g039260, Csa09g061550) whereas CsPALlike2 detects two PAL4 homologs (Csa01g011180, Csa15g012820). The expression levels of Cs-PAL-like1 and CsPAL-like2 were not statistically different in the transgenic lines from those of wild type plants (Figure 4B) suggesting that factors other than transcriptional regulation are suppressing PAL activity in the transgenic plants. The Arabidopsis study showed that PAL activity is affected by its stability through the $26 \mathrm{~S}$ proteasome-mediated degradation (Zhang et al., 2013). Once PALs are ubiquitinated, the poly-ubiquitinated PALs are degraded by $26 \mathrm{~S}$ proteasome. A specific group of kelch-domain F-Box proteins (KFBs) are involved in the PAL ubiquitination in Arabidopsis (Zhang et al., 2013; Zhang et al., 2015). These KFBs are components of ubiquitin E3 ligase, and AtKFB1, AtKFB20, AtKFB39, and AtKFB50 facilitate ubiquitination of PAL redundantly (Zhang et al., 2013; Zhang et al., 2015). In Arabidopsis, the accumulation of IAOx or its derivatives enhances the expression of these four KFBs and in turn, results in reduced PAL activity and phenylpropanoid production (Kim et al., 2019). When AtKFBs are disrupted in high aldoximecontaining mutants, phenylpropanoid deficiency is restored (Kim et al., 2019). Thus, we hypothesized that a similar mechanism may function in the repression of PAL in C. sativa.

To identify Camelina sativa KFB homologs, we did phylogenetic analysis for KFB homologs using the $C$. sativa-annotated protein dataset with Arabidopsis KFB1 (Atlg15670), KFB20 (Atlg80440), KFB39 (At2g44130), and KFB50 (At3g59940). A total of 459 transcript models containing kelch-motif(s) were identified using a Hidden Markov Model (HMM) search (Figure 5A). Close homologs of AtKFB1/20/39/50 were selected: Csa17g021220, Csa03g019430, and Csa14g018700 are homologs of At1g15670/ KFB1; Csa09g094570, and Csa07g059430 for At1g80440/KFB20; Csa06g050450, Csa05g006160, and Csa04g061960 for At2g44130/ KFB39; Csa07g005130, Csa05g091560, Csa16g006020 for At3g59940/KFB50 (Figure 5B). Each group of Camelina KFB homologs has $77 \%$ to $89 \%$ amino acid sequence identity with Arabidopsis KFBs (Table S2).

Since CsKFB50-like genes were most abundant in our samples as shown in Table S3, we cloned Csa05g091560, one of the AtKFB50 homologs, to test its function. As shown in Figure 6, the expression of Csa05g091560 in Arabidopsis was negatively correlated with PAL activity and produced lower levels of phenylpropanoids (Figures 6A, B). However, the transgenic lines with highly expressed Csa05g091560 displayed stunted growth and less purple color, indicating anthocyanin deficiency (Figure 6C). Dwarfism and phenylpropanoid deficiency together with reduced PAL activity in the transgenic lines mimicked phenotypes in Arabidopsis pal1/2/4 mutants and 

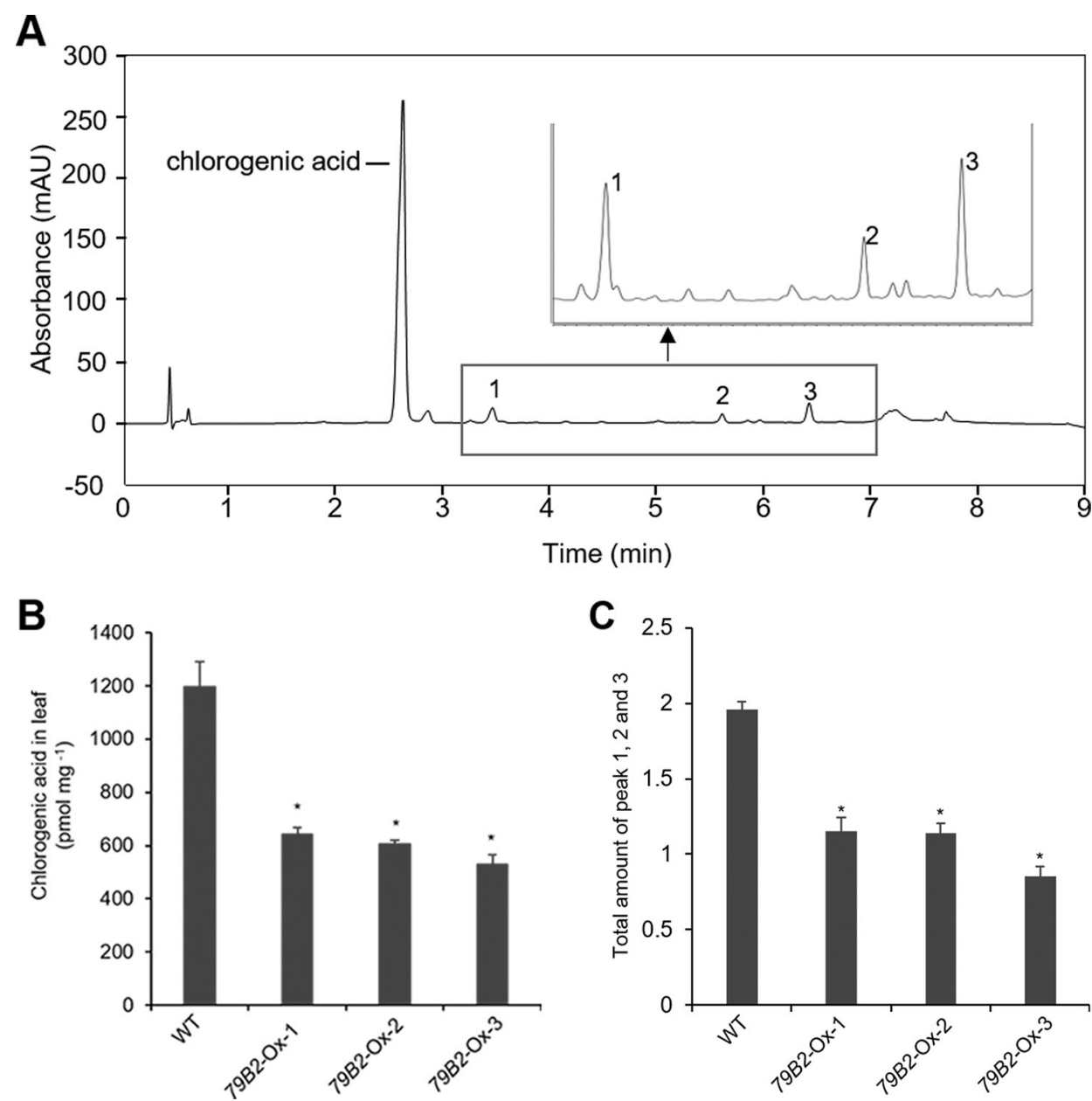

FIGURE 3 | Overexpression of AtCYP79B2 inhibits phenylpropanoid production in Camelina.Representative chromatogram of soluble metabolites in wild-type Camelina leaves is shown (A). The extract gave four recognizable peaks at $325 \mathrm{~nm}$ and chlorogenic acid is a major peak. The inset shows three peaks eluted between 3 and 7 min. Chlorogenic acid contents (B) and the total sum of peak 1, 2, and 3 (C) of the AtCYP79 overexpression lines compared with wild type are shown. Leaf samples were collected from 2-week-old soil grown plants. The total amount of peak 1 to 3 was calculated based on the peak area of each peak. In (B) and $\mathbf{( C )}$, data represent mean \pm SE of three independent experiments. * represents $P<0.05$ (two-tailed student's t-test) when compared with wild type.

$A t K F B$ overexpression lines, which suggests its possible roles on PAL degradation similar to the Arabidopsis KFBs. (Huang et al., 2010; Zhang et al., 2013; Zhang et al., 2015).

\section{The Expression of CsKFBs Functioning in PAL Degradation Is Increased in the AtCYP79B2 Overexpression Lines}

To evaluate any impact of the altered aldoxime production on the expression of $C s K F B s$, we analyzed the expression levels of these CsKFBs in the transgenic lines. We designed four sets of primers using their conserved sequences. CsKFB1-like can detect all three AtKFB1 homologs (Csa17g021220, Csa03g019430, Csa14g018700). Similarly, CsKFB20-like, CsKFB39-like, and CsKFB50-like detect two AtKFB20 homologs (Csa09g094570, Csa07g059430), three AtKFB39 homologs (Csa06g050450, Csa05g006160, Csa04g061960), and three AtKFB50 homologs (Csa07g005130, Csa05g091560, Csa16g006020), respectively
(Table S2). As shown in Figure 7A, the expression of $C s K F B$ genes was significantly increased in the AtCYP79B2 overexpression lines compared with that of wild-type plants.

\section{DISCUSSION}

\section{Overexpression of AtCYP79B2 Increases Auxin Contents But Does Not Affect Indole Glucosinolate Production in Camelina}

IAA a major auxin in plants is made mainly through the YUC pathway, the common auxin biosynthesis pathway. The YUC pathway is a simple two-step biosynthesis pathway using two enzymes, TAA (Tryptophan Aminotransferase of Arabidopsis) and YUCCA (Flavin containing monooxygenase) (Bak and Feyereisen, 2001; Kim et al., 2007; Dai et al., 2013; Korasick et al., 2013). In addition to the YUC pathway, IAA can be made 

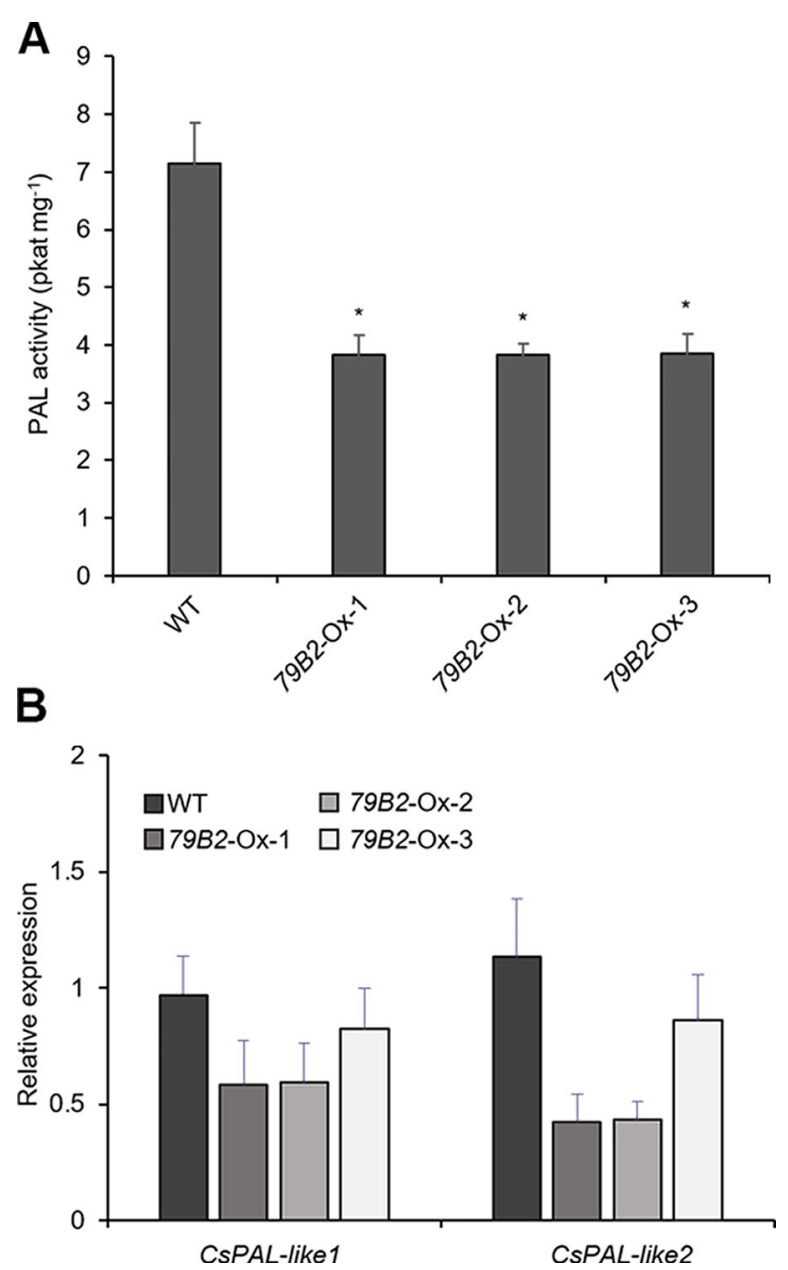

FIGURE 4 | Phenylalanine ammonia lyase (PAL) activity is reduced in AtCYP79B2 overexpression lines. (A) PAL activities were measured using crude extracts of leaves from 2-week-old soil grown Camelina plants. (B) Transcript abundance of Camelina PALs in transgenic lines and wild type was measured with quantitative RT-PCR. CsPAL-like1 represents expression of six genes (Csa06g039710, Csa05g016810, Csa04g050600, Csa06g027730, Csa04g039260, and Csa09g061550) which are Arabidopsis PAL1 and PAL2 homologs. CsPAL-like2 represents expression of Csa01g011180 and Csa15g012820, which are Arabidopsis PAL4 homologs. In (A) and $(\mathbf{B})$, data represent mean \pm SE of three independent experiments. * represents $P<0.05$ (two-tailed student's t-test) when compared with wild type.

from IAOx in Arabidopsis under stress conditions (Zhao et al., 2002; Nonhebel et al., 2011). Overexpression of CYP79B2encoding IAOx producing enzyme, or disruption of the IAOxconsuming enzyme CYP83B1 results in the increased levels of IAA in Arabidopsis, indicating the production of IAA from IAOx (Delarue et al., 1998; Barlier et al., 2000; Zhao et al., 2002; Sugawara et al., 2009). Our data suggest that the IAOxdependent IAA biosynthesis pathway is conserved in Camelina as well. It was previously shown that CsCYP79Bs, Csa11g003080, and Csa10g002730 were coexpressed with other genes encoding auxin transporters and auxin biosynthesis enzymes in response

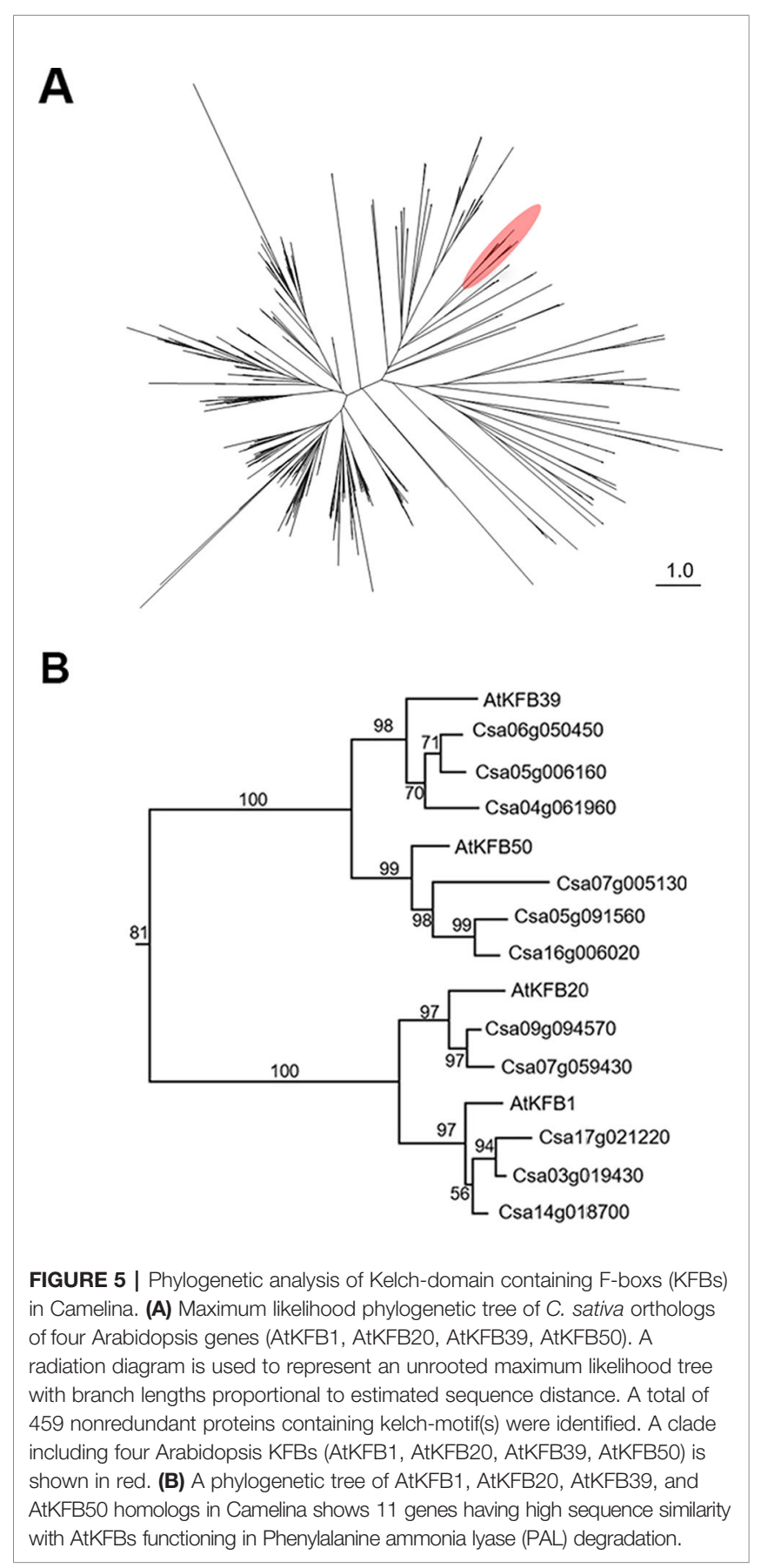

to stresses (Heydarian et al., 2018). It is possible that the IAOxderived IAA production in Camelina is relevant to stress-induced growth modulation as shown in Arabidopsis (Franklin et al., 2011).

Previous studies have shown that Arabidopsis plants overexpressing CYP79B contain increased indole glucosinolates (Zhao et al., 2002). However, we were not able to detect indole glucosinolates in young seedlings or old leaves of both Camelina wild type and all three overexpression lines (Figure 2B). Over 30 different structures of glucosinolates are found in Arabidopsis 


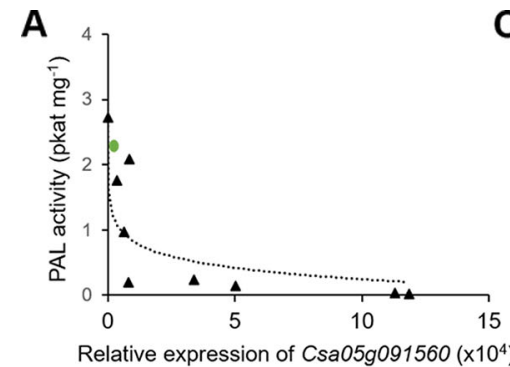

B
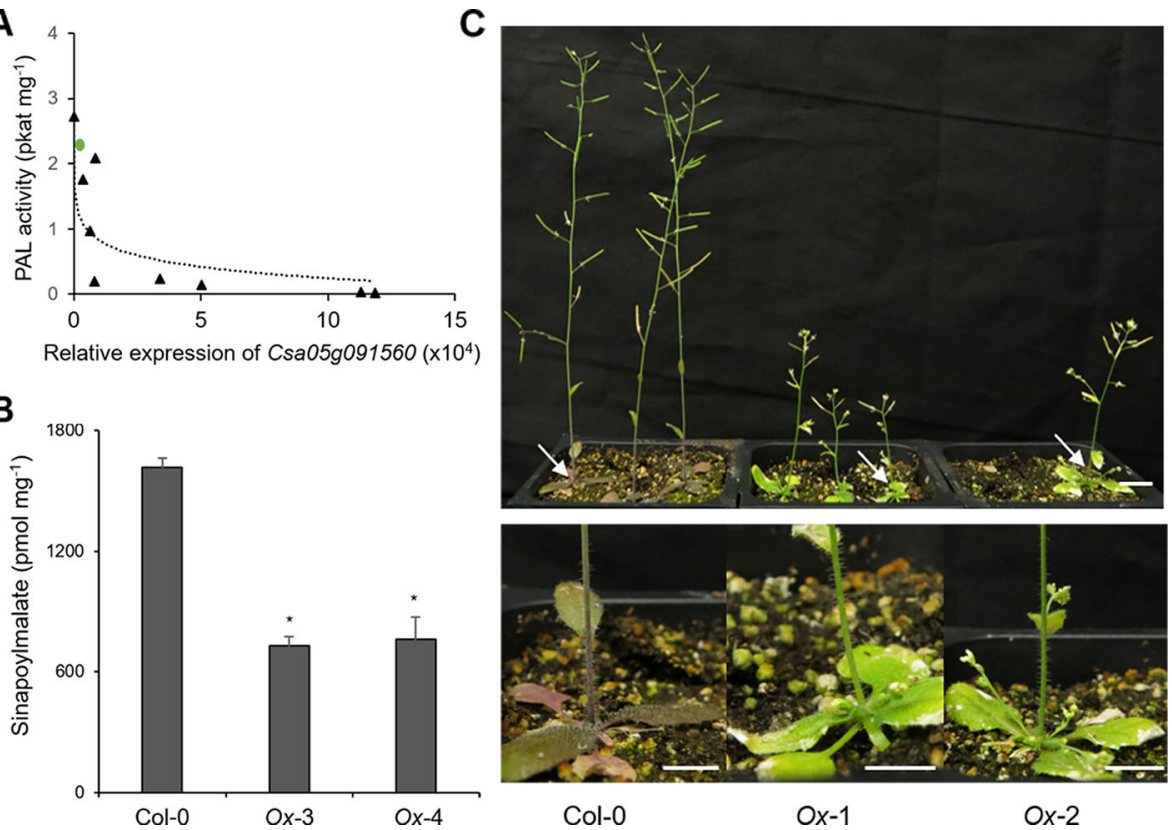

FIGURE 6 | Overexpression of Csa05g091560, one of the CsKFB50-like genes,reduces Phenylalanine ammonia lyase (PAL) activity and inhibits phenylpropanoid production in Arabidopsis. (A) PAL activity of nine Arabidopsis transgenic lines overexpressing Csa05g091560 is negatively correlated with the expression of Csa05g091560. Green circle indicates PAL activity of Arabidopsis wild type. (B) Sinapoylmalate contents in Arabidopsis plants overexpressing Csa05g091560. Soluble metabolites were extracted from 3-week-old plants grown on soil. Data represent mean \pm SE of three independent experiments. ${ }^{*}$ represents $p<0.05$ (twotailed student's t-test) when compared with wild type. (C) Stunted growth and reduced anthocyanin accumulation of two transgenic lines overexpressing Csa05g091560 are shown compared with control plants (Col-0). Close-up images of plants show anthocyanin deficiency in the transgenic lines marked with arrows. (bars $=1 \mathrm{~cm}$ ) Photos were taken five weeks after planting.

and their profiles vary depending on organs and ecotypes (Kliebenstein et al., 2001). Camelina seeds accumulate aliphatic glucosinolates including glucoarabin (9-(methylsulfinyl) nonylglucosinolate), glucocamelinin (10-(methylsulfinyl) decylglucosinolate), and 11-(methylsulfinyl)undecylglucosinolate (Berhow et al., 2013; Das et al., 2014; Russo et al., 2014). The $C$. sativa genome study identified that the $C$. sativa genome represents a whole-genome triplication event relative to the Arabidopsis thaliana genome (Kagale et al., 2014). Indeed, we found that CYP79Bs and KFBs of Arabidopsis and Camelina have high sequence identities (Table 1; Table S2). But, the glucosinolate profiles in Camelina are quite different from those of Arabidopsis. It is possible that other factors such as specific pest infestation or pathogen attack are required to activate glucosinolate production. Apparently, IAOx is not a limiting factor for Camelina to produce indole glucosinolates, at least in seedlings and leaves.

\section{Altered IAOx Metabolism Affects Phenylpropanoid Production Through Transcriptional Activation of Genes Functioning in PAL Degradation in Camelina}

The fact that the expression of PALs is not altered while these $C s K F B$-like genes are up-regulated in the AtCYP79B2 overexpression lines suggests that increased $C s K F B$ expression can contribute to reduced PAL activity as well as the reduction of phenylpropanoids in the transgenic lines. The activation of $K F B$ expression and reduced PAL activity in the Camelina transgenic lines are reminiscent of a molecular mechanism of phenylpropanoid repression in Arabidopsis high-aldoxime mutants (Kim et al., 2019). The biosynthetic pathway of phenylpropanoids and its regulation have been extensively studied. Studies have revealed that the phenylpropanoid pathway can be regulated at various levels. Transcription factors or general transcription machineries such as Mediator subunit 5 (MED5) are involved in the expression of genes encoding biosynthetic enzymes (Bonawitz et al., 2012; Dolan et al., 2017). Feedforward or feedback regulations and posttranslational modification of enzymes such as phosphorylation or ubiquitination play roles in the activity or stability of enzymes (Zhang et al., 2013; Zhang et al., 2015). In addition, the protein-protein interaction is critical to form the lignin metabolon (Gou et al., 2018). Our data presented here and the previous Arabidopsis studies indicate that phenylpropanoid metabolism can be affected by the status of other metabolite biosynthetic pathways (Figure 7B). Since PAL functions at the entry point of the phenylpropanoid pathway, the influence of aldoxime metabolism on PAL stability can affect the entire phenylpropanoid production. It appears that the molecular mechanism behind the link between aldoxime metabolism and phenylpropanoid production is conserved between Arabidopsis and Camelina. It was shown that the content of glucosinolates is 


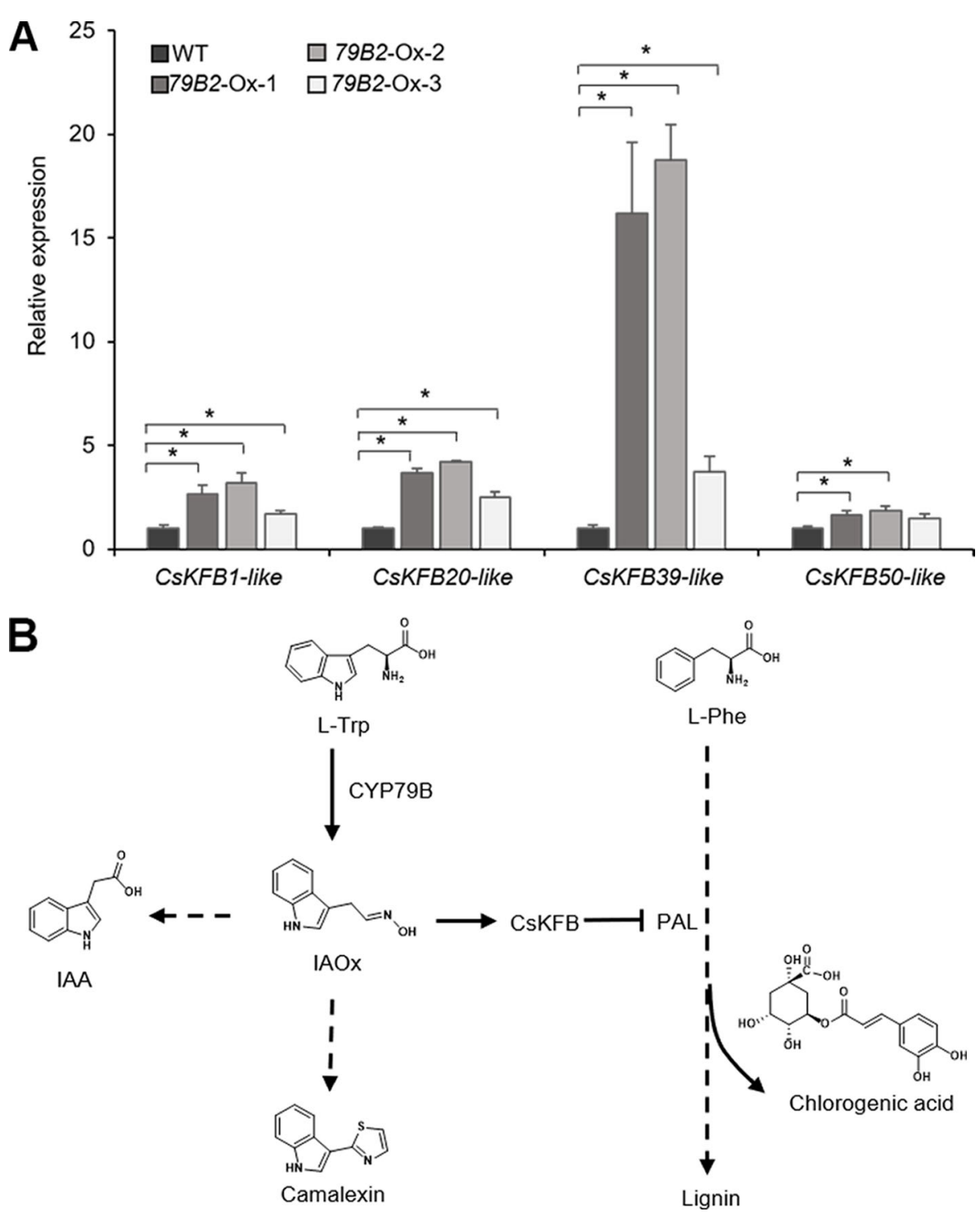

FIGURE 7 | Increased expression of CSKFBs in the Camelina transgenic plants and a proposed model showing a link between IAOx metabolism and the phenylpropanoid pathway in Camelina. (A) Transcript abundance of CsKFBs in Camelina transgenic lines overexpressing AtCYP79B2. CsKFB1-like represents the expression of three KFB1 homolog genes (Csa17g021220, Csa03g019430, Csa14g018700). CsKFB20-like represents two CsKFB20-like genes (Csa09g094570, Csa07g059430). CsKFB39-like and CsKFB50-like represent three CsKFB39-like genes (Csa06g050450, Csa05g006160, Csa04g061960) and three CsKFB50-like genes (Csa07g005130, Csa05g091560, Csa16g006020), respectively. Data represent mean \pm SE $(n=3)$. * represents $P<0.05$ (two-tailed student's t-test) when compared with wild type. (B) A proposed model demonstrating that increased IAOx production influences the expression of CsKFBs functioning in PAL degradation, thereby affecting the phenylpropanoid production in Camelina.

inversely correlated with the level of sinapine (Russo and Reggiani, 2012). It is possible that the negative correlation between the sinapine content and the glucosinolate content in Camelina may be explained by the phenylpropanoid repression by aldoxime metabolism. It is worth noting that Arabidopsis produces indole glucosinolates from IAOx but Camelina does not although both produce IAA and camalexin from IAOx. This result further confirms that aldoximes or their derivatives, not glucosinolates, are key molecules affecting transcriptional activation of KFBs. Recent studies have identified aldoximeproducing enzymes in various species including monocots and gymnosperms (Irmisch et al., 2013; Irmisch et al., 2015; Luck et al., 2017; Bhalla et al., 2018; Dhandapani et al., 2019). Considering that aldoxime metabolism is widely spread in the plant kingdom, this type of link may exist in species beyond Brassicales. 


\section{DATA AVAILABILITY STATEMENT}

Sequence of genes and proteins in this study can be found in the GenBank under the following accession numbers: AtCYP79B2 (At4g39950), AtCYP79B3 (At2g22330), AtKFB1 (At1g15670), AtKFB20 (At1g80440), AtKFB39 (At2g44130), AtKFB50 (At3g59940), Csa10g002730 (XP_019086033), Csa12g003200 (XP_010446588), Csa12g002890 (XP_010446562), Csa11g003080 (XP_010437120), Csa7g052430 (XP_010416994), Csa16g044070 (XP_010472222), Csa9g086670 (XP_010429154), Csa06g050450 (XP_010518004), Csa05g006160 (XP_010508332), Csa04g061960 (XP_010506327), Csa07g005130 (XP_010413616), Csa05g091560 (XP_010512288), Csa16g006020 (XP_010469249), Csa09g094570 (XP_010417707), Csa07g059430 (XP_010429956), Csa17g021220 (XP_010476655), Csa03g019430 (XP_010497299), Csa14g018700 (XP_010459104), Csa06g039710 (XP_010516949), Csa05g016810 (XP_010509401), Csa04g050600 (XP_010505261), Csa06g027730 (XP_010515816), Csa04g039260 (XP_010504083), Csa09g061550 (XP_010426963), Csa01g011180 (XP_010482945), Csa15g012820 (XP_010464706).

\section{AUTHOR CONTRIBUTIONS}

$\mathrm{DZ}$ and $\mathrm{JK}$ conceived and designed the experiments. DZ, YS, and RD performed the experiments. TL generated phylogenetic trees. $\mathrm{DZ}$ and JK wrote the manuscript. All authors read and approved the final manuscript.

\section{REFERENCES}

Agerbirk, N., and Olsen, C. E. (2012). Glucosinolate structures in evolution. Phytochemistry 77, 16-45. doi: 10.1016/j.phytochem.2012.02.005

Bak, S., and Feyereisen, R. (2001). The Involvement of Two P450 Enzymes, CYP83B1 and CYP83A1, in Auxin Homeostasis and Glucosinolate Biosynthesis. Plant Physiol. 127, 108-118. doi: 10.1104/pp.127.1.108

Barlier, I., Kowalczyk, M., Marchant, A., Ljung, K., Bhalerao, R., Bennett, M., et al. (2000). The SUR2 gene of Arabidopsis thaliana encodes the cytochrome P450 CYP83B1, a modulator of auxin homeostasis. PNAS 97, 14819-14824. doi: 10.1073/pnas.260502697

Berhow, M. A., Polat, U., Glinski, J. A., Glensk, M., Vaughn, S. F., Isbell, T., et al. (2013). Optimized analysis and quantification of glucosinolates from Camelina sativa seeds by reverse-phase liquid chromatography. Ind. Crops Prod. 43, 119125. doi: $10.1016 /$ j.indcrop.2012.07.018

Bhalla, T. C., Kumar, V., and Kumar, V. (2018). Enzymes of aldoxime-nitrile pathway for organic synthesis. Rev. Environ. Sci. Biotechnol. 17, 229-239. doi: 10.1007/s11157-018-9467-0

Bonawitz, N. D., Soltau, W. L., Blatchley, M. R., Powers, B. L., Hurlock, A. K., Seals, L. A., et al. (2012). REF4 and RFR1, Subunits of the Transcriptional Coregulatory Complex Mediator, Are Required for Phenylpropanoid Homeostasis in Arabidopsis. J. Biol. Chem. 287, 5434-5445. doi: 10.1074/jbc.M111.312298

Browne, L. M., Conn, K. L., Ayert, W. A., and Tewari, J. P. (1991). The camalexins: New phytoalexins produced in the leaves of Camelina sativa (cruciferae). Tetrahedron 47, 3909-3914. doi: 10.1016/S0040-4020(01)86431-0

Chen, S., Glawischnig, E., Jørgensen, K., Naur, P., Jørgensen, B., Olsen, C.-E., et al. (2003). CYP79F1 and CYP79F2 have distinct functions in the biosynthesis of aliphatic glucosinolates in Arabidopsis. Plant J. 33, 923-937. doi: 10.1046/ j.1365-313X.2003.01679.x

Dai, X., Mashiguchi, K., Chen, Q., Kasahara, H., Kamiya, Y., Ojha, S., et al. (2013). The biochemical mechanism of auxin biosynthesis by an Arabidopsis YUCCA flavin-containing monooxygenase. J. Biol. Chem. 288, 1448-1457. doi: 10.1074/ jbc.M112.424077

\section{FUNDING}

This work was supported by USDA NIFA Hatch (005681) and a startup fund from the Horticultural Sciences Department and Institute of Food and Agricultural Sciences at the University of Florida. This work was also supported by the Principal Investigator of Postdoctoral Fellowship Program through the National Research Foundation of Korea (NRF) grant funded by the Ministry of Education (No.2018R1A6A3A03013074, YS, Republic of Korea).

\section{ACKNOWLEDGMENTS}

We thank Dr. Jeff Harper at the University of Nebraska for sharing Camelina sativa cv. Celine seeds. We thank ICBR Proteomics \& Mass Spectrometry with LC-MS operation.

\section{SUPPLEMENTARY MATERIAL}

The Supplementary Material for this article can be found online at: https:/www.frontiersin.org/articles/10.3389/fpls.2020.00017/ full\#supplementary-material

Das, N., Berhow, M. A., Angelino, D., and Jeffery, E. H. (2014). Camelina sativa defatted seed meal contains both alkyl sulfinyl glucosinolates and quercetin that synergize bioactivity. J. Agric. Food Chem. 62, 8385-8391. doi: 10.1021/ jf501742h

Delarue, M., Prinsen, E., Onckelen, V. H., Caboche, M., and Bellini, C. (1998). Sur2 mutations of Arabidopsis thaliana define a new locus involved in the control of auxin homeostasis. Plant J. 14, 603-611. doi: 10.1046/j.1365313X.1998.00163.x

Dhandapani, S., Jin, J., Sridhar, V., Chua, N.-H., and Jang, I.-C. (2019). CYP79D73 participates in biosynthesis of floral scent compound 2-phenylethanol in Plumeria rubra. Plant Physiol. 180 (1), 171-184. doi: 10.1104/pp.19.00098

Dolan, W. L., Dilkes, B. P., Stout, J. M., Bonawitz, N. D., and Chapple, C. (2017). Mediator Complex Subunits MED2, MED5, MED16, and MED23 Genetically Interact in the Regulation of Phenylpropanoid Biosynthesis. Plant Cell 29, 3269-3285. doi: 10.1105/tpc.17.00282

Edgar, R. C. (2004). MUSCLE: multiple sequence alignment with high accuracy and high throughput. Nucleic Acids Res. 32, 1792-1797. doi: 10.1093/nar/gkh340

Franklin, K. A., Lee, S. H., Patel, D., Kumar, S. V., Spartz, A. K., Gu, C., et al. (2011). PHYTOCHROME-INTERACTING FACTOR 4 (PIF4) regulates auxin biosynthesis at high temperature. PNAS 108, 20231-20235. doi: 10.1073/ pnas. 1110682108

Fraser, C. M., and Chapple, C. (2011). The Phenylpropanoid Pathway in Arabidopsis. Arabidopsis Book 9, e0152. doi: 10.1199/tab.0152

Gou, M., Ran, X., Martin, D. W., and Liu, C.-J. (2018). The scaffold proteins of lignin biosynthetic cytochrome P450 enzymes. Nat. Plants 4, 299. doi: 10.1038/ s41477-018-0142-9

Hansen, C. H., Wittstock, U., Olsen, C. E., Hick, A. J., Pickett, J. A., and Halkier, B. A (2001). Cytochrome P450 CYP79F1 from Arabidopsis Catalyzes the Conversion of Dihomomethionine and Trihomomethionine to the Corresponding Aldoximes in the Biosynthesis of Aliphatic Glucosinolates. J. Biol. Chem. 276, 11078-11085. doi: 10.1074/jbc.M010123200

Hemm, M. R., Ruegger, M. O., and Chapple, C. (2003). The Arabidopsis ref2 mutant is defective in the gene encoding CYP83A1 and shows both 
phenylpropanoid and glucosinolate phenotypes. Plant Cell 15, 179-194. doi: $10.1105 /$ tpc.006544

Heydarian, Z., Yu, M., Gruber, M., Coutu, C., Robinson, S. J., and Hegedus, D. D. (2018). Changes in gene expression in Camelina sativa roots and vegetative tissues in response to salinity stress. Sci. Rep. 8, 9804. doi: 10.1038/s41598-018-28204-4

Huang, J., Gu, M., Lai, Z., Fan, B., Shi, K., Zhou, Y.-H., et al. (2010). Functional Analysis of the Arabidopsis PAL Gene Family in Plant Growth, Development, and Response to Environmental Stress. Plant Physiol. 153, 1526-1538. doi: $10.1104 /$ pp. 110.157370

Irmisch, S., McCormick, A. C., Boeckler, G. A., Schmidt, A., Reichelt, M., Schneider, B., et al. (2013). Two Herbivore-Induced Cytochrome P450 Enzymes CYP79D6 and CYP79D7 Catalyze the Formation of Volatile Aldoximes Involved in Poplar Defense. Plant Cell 25, 4737-4754. doi: 10.1105/tpc.113.118265

Irmisch, S., Zeltner, P., Handrick, V., Gershenzon, J., and Köllner, T. G. (2015). The maize cytochrome P450 CYP79A61 produces phenylacetaldoxime and indole-3acetaldoxime in heterologous systems and might contribute to plant defense and auxin formation. BMC Plant Biol. 15, 128. doi: 10.1186/s12870-015-0526-1

Kagale, S., Koh, C., Nixon, J., Bollina, V., Clarke, W. E., Tuteja, R., et al. (2014). The emerging biofuel crop Camelina sativa retains a highly undifferentiated hexaploid genome structure. Nat. Commun. 5, 3706. doi: 10.1038/ ncomms 4706

Kim, J. I., Sharkhuu, A., Jin, J. B., Li, P., Jeong, J. C., Baek, D., et al. (2007). yucca6, a Dominant Mutation in Arabidopsis, Affects Auxin Accumulation and Auxin-Related Phenotypes. Plant Physiol. 145, 722-735. doi: 10.1104/ pp.107.104935

Kim, J. I., Dolan, W. L., Anderson, N. A., and Chapple, C. (2015). Indole Glucosinolate Biosynthesis Limits Phenylpropanoid Accumulation in Arabidopsis thaliana. Plant Cell 27, 1529-1546. doi: 10.1105/tpc.15.00127

Kim, J. I., Zhang, X., Pascuzzi, P. E., Liu, C.-J., and Chapple, C. (2019). Glucosinolate and phenylpropanoid biosynthesis are linked by proteasome-dependent degradation of PAL. New Phytol. 225 (1), 154-168 doi: 10.1111/nph.16108

Klein, A. P., Anarat-Cappillino, G., and Sattely, E. S. (2013). Minimum Set of Cytochromes P450 for Reconstituting the Biosynthesis of Camalexin, a Major Arabidopsis Antibiotic. Angew. Chem. 125, 13870-13873. doi: 10.1002/ ange.201307454

Kliebenstein, D. J., Kroymann, J., Brown, P., Figuth, A., Pedersen, D., Gershenzon, J., et al. (2001). Genetic Control of Natural Variation in Arabidopsis Glucosinolate Accumulation. Plant Physiol. 126, 811-825. doi: 10.1104/pp.126.2.811

Korasick, D. A., Enders, T. A., and Strader, L. C. (2013). Auxin biosynthesis and storage forms. J. Exp. Bot. 64, 2541-2555. doi: 10.1093/jxb/ert080

Lee, S., Kaminaga, Y., Cooper, B., Pichersky, E., Dudareva, N., and Chapple, C. (2012). Benzoylation and sinapoylation of glucosinolate R-groups in Arabidopsis. Plant J. 72, 411-422. doi: 10.1111/j.1365-313X.2012.05096.x

Lu, C., and Kang, J. (2008). Generation of transgenic plants of a potential oilseed crop Camelina sativa by Agrobacterium-mediated transformation. Plant Cell Rep. 27, 273-278. doi: 10.1007/s00299-007-0454-0

Luck, K., Jia, Q., Huber, M., Handrick, V., Wong, G. K.-S., Nelson, D. R., et al. (2017). CYP79 P450 monooxygenases in gymnosperms: CYP79A118 is associated with the formation of taxiphyllin in Taxus baccata. Plant Mol. Biol. 95, 169-180. doi: 10.1007/s11103-017-0646-0

Malik, M. R., Tang, J., Sharma, N., Burkitt, C., Ji, Y., Mykytyshyn, M., et al. (2018). Camelina sativa, an oilseed at the nexus between model system and commercial crop. Plant Cell Rep. 37, 1367-1381. doi: 10.1007/s00299-018-2308-3

Mikkelsen, M. D., Hansen, C. H., Wittstock, U., and Halkier, B. A. (2000). Cytochrome P450 CYP79B2 from Arabidopsis Catalyzes the Conversion of Tryptophan to Indole-3-acetaldoxime, a Precursor of Indole Glucosinolates and Indole-3-acetic Acid. J. Biol. Chem. 275, 33712-33717. doi: 10.1074/ jbc.M001667200

Naur, P., Petersen, B. L., Mikkelsen, M. D., Bak, S., Rasmussen, H., Olsen, C. E., et al. (2003). CYP83A1 and CYP83B1, Two Nonredundant Cytochrome P450
Enzymes Metabolizing Oximes in the Biosynthesis of Glucosinolates in Arabidopsis. Plant Physiol. 133, 63-72. doi: 10.1104/pp.102.019240

Nonhebel, H., Yuan, Y., Al-Amier, H., Pieck, M., Akor, E., Ahamed, A., et al. (2011). Redirection of tryptophan metabolism in tobacco by ectopic expression of an Arabidopsis indolic glucosinolate biosynthetic gene. Phytochemistry 72, 37-48. doi: 10.1016/j.phytochem.2010.10.018

Novák, O., Hényková, E., Sairanen, I., Kowalczyk, M., Pospíšil, T., and Ljung, K. (2012). Tissue-specific profiling of the Arabidopsis thaliana auxin metabolome. Plant J. 72, 523-536. doi: 10.1111/j.1365-313X.2012.05085.x

Ruegger, M., and Chapple, C. (2001). Mutations That Reduce Sinapoylmalate Accumulation in Arabidopsis thaliana Define Loci With Diverse Roles in Phenylpropanoid Metabolism. Genetics 159, 1741-1749.

Russo, R., and Reggiani, R. (2012). Antinutritive Compounds in Twelve Camelina sativa Genotypes. Am. J. Plant Sci. 03, 1408-1412. doi: 10.4236/ ajps.2012.310170

Russo, R., Galasso, I., and Reggiani, R. (2014). Variability in glucosinolate content among Camelina species. Am. J. Plant Sci. 5, 294-298. doi: 10.4236/ ajps.2014.53040

Sørensen, M., Neilson, E. H. J., and Møller, B. L. (2018). Oximes: Unrecognized Chameleons in General and Specialized Plant Metabolism. Mol. Plant 11, 95117. doi: 10.1016/j.molp.2017.12.014

Stamatakis, A. (2014). RAxML version 8: a tool for phylogenetic analysis and postanalysis of large phylogenies. Bioinformatics 30, 1312-1313. doi: 10.1093/ bioinformatics/btu033

Sugawara, S., Hishiyama, S., Jikumaru, Y., Hanada, A., Nishimura, T., Koshiba, T., et al. (2009). Biochemical analyses of indole-3-acetaldoxime-dependent auxin biosynthesis in Arabidopsis. PNAS 106, 5430-5435. doi: 10.1073/ pnas.0811226106

Vogt, T. (2010). Phenylpropanoid biosynthesis. Mol. Plant 3, 2-20. doi: 10.1093/ $\mathrm{mp} / \mathrm{ssp} 106$

Weng, J.-K., Mo, H., and Chapple, C. (2010). Over-expression of F5H in COMTdeficient Arabidopsis leads to enrichment of an unusual lignin and disruption of pollen wall formation. Plant J. 64, 898-911. doi: 10.1111/j.1365313X.2010.04391.x

Wittstock, U., and Halkier, B. A. (2000). Cytochrome P450 CYP79A2 from Arabidopsis thaliana L. Catalyzes the Conversion of 1-Phenylalanine to Phenylacetaldoxime in the Biosynthesis of Benzylglucosinolate. J. Biol. Chem. 275, 14659-14666. doi: 10.1074/jbc.275.19.14659

Zhang, X., Gou, M., and Liu, C.-J. (2013). Arabidopsis Kelch Repeat F-Box Proteins Regulate Phenylpropanoid Biosynthesis via Controlling the Turnover of Phenylalanine Ammonia-Lyase. Plant Cell 25, 4994-5010. doi: 10.1105/tpc.113.119644

Zhang, X., Gou, M., Guo, C., Yang, H., and Liu, C.-J. (2015). Down-Regulation of Kelch Domain-Containing F-Box Protein in Arabidopsis Enhances the Production of (Poly)phenols and Tolerance to Ultraviolet Radiation. Plant Physiol. 167, 337-350. doi: 10.1104/pp.114.249136

Zhao, Y., Hull, A. K., Gupta, N. R., Goss, K. A., Alonso, J., Ecker, J. R., et al. (2002). Trp-dependent auxin biosynthesis in Arabidopsis: involvement of cytochrome P450s CYP79B2 and CYP79B3. Genes Dev. 16, 3100-3112. doi: 10.1101/ gad.1035402

Conflict of Interest: The authors declare that the research was conducted in the absence of any commercial or financial relationships that could be construed as a potential conflict of interest.

Copyright (c) 2020 Zhang, Song, Dai, Lee and Kim. This is an open-access article distributed under the terms of the Creative Commons Attribution License (CC BY). The use, distribution or reproduction in other forums is permitted, provided the original author(s) and the copyright owner(s) are credited and that the original publication in this journal is cited, in accordance with accepted academic practice. No use, distribution or reproduction is permitted which does not comply with these terms. 